\title{
Clustered multidimensional data association for limited sensor resolutions
}

\author{
Felix Opitz \\ Defence and Communication Systems / Air and Naval Defence \\ EADS Deutschland GmbH \\ Wörthstr. 85, 89077 Ulm, Germany. \\ felix.opitz@eads.com
}

\begin{abstract}
The multidimensional data association methods were developed to establish the relation between measurements and tracks especially in dense target situations. However, even these advanced multidimensional data association methods lack in situations of unresolved measurement. Specifically, in real dense target situations being of most interest the phenomena of unresolved measurements happens quite often due to the limited sensor resolution.
\end{abstract}

The new algorithm presented in this paper incorporates the unresolved measurement hypothesis into the multidimensional data association approach. An additional feature of this approach is, that also group tracking aspects are considered, which is an essential difference to proposals of other authors. Therefore, this new approach significantly increases the data association result as well as track accuracy, continuity and ensures early track initialisation capabilities.

Keywords: Tracking, data association, unresolved measurements, optimization theory, Linear Programming, group tracking, clustering.

\section{Introduction}

The data association describes the correlation between tracks representing targets and plots found by a single sensor or a sensor suite. Therefore, one has to distinguish between several hypotheses about the origin of a new sensor measurement: It may be unreal and has to be ignored (false alarm). Otherwise, it is assumed to be real and then one has to discriminate between measurements related to tracks supported by previous measurements (update) and those, which are not (new track). Finally, an existing track may be not reported due to a miss (no detection). I.e., the standard algorithms distinguish between the following hypotheses:

- False alarm / new track,

- $\quad$ Measured plot of an established track

- No detection
Up to now, there is a wide spectrum of algorithms addressed to this problem.

The Joint Probabilistic Data Association method attempts to find an association between plots and targets in a probabilistic fashion. This is to be distinguished from hard decision methods. The easiest of them is the nearest neighbour approach, which establishes a relation between a plot and the track having the shortest distance. Multidimensional data association collects plots into so called scans, which may be identified with a full turn of a mechanically scanning radar. The collection of plots into several scans implies constraints for the data association problem:

- Each plot of a scan is associated with at most one track and

- $\quad$ Each track is associated with at most one plot.

Multidimensional data association attempts to maximize the association probability subject to above constraints. Therefore, the data association problem is translated into a linear integer optimisation problem with constraints. Considering the association between the existing tracks and the measurements of $\mathrm{N}-1$ further scans define an $\mathrm{N}$ dimensional data association problem.

The algorithmic realisation of a two dimensional data association can be solved by the Auction [1, 2], the Jonker-Volgenant-Castanon [3], or the more classical Munkres algorithm [4]. Unfortunately, the higher dimensional association problems $(\mathrm{N}>2)$ are $\mathrm{NP}$ hard problems.

One method to overcome the complexity problems uses Lagrange Relaxation to remove the above constraints [5, 6]. The optimisation problem is reduced to a convex optimisation problem in the Lagrange multipliers. This is handled by non differentiable optimisation theory e.g. recently found bundle trust methods [7, 8, 9], which offer a very stable iteration scheme. The result found is an integer and feasible one with respect to the above constraints, but the price is that this method is suboptimal. 
A Linear Programming approach [10] uses the Homogeneous Self-Dual Interior Point Method [11, 12] to solve the optimisation problem. The found solution is an optimal one but needs not to be an integer one. Therefore, the solution found requires an additional interpretation as pseudo probabilities, which can be given through a statistical view which is similar to the one used by the Joint Probabilistic Data Association technique.

However, the lack of all above methods is the ignorance of the limited sensor resolution, which causes unresolved measurements. The common occurrence of those phenomena within any real radar environment has the potential to decrease the performance of above algorithms in both track continuity and track accuracy dramatically. So, the impact of unresolved measurements for tracking applications were studied by several authors. In [13] the JPDA approach is enriched by the unresolved measurement and a probability model for merged plots is derived. In [14] W. Koch and G. van Keuk consider unresolved measurements together with group tracking aspects within a classical MHT approach. H. Chen, T. Kirubarajan, and Y. Bar-Shalom [15] improve the behaviour by modifying the constraints of the data association method to allow also one-to-n association between the tracks and the measurements which fall into one scan, but did not take into account group tracking aspects.

There are two opposite approaches of group tracking in the literature [16] according to different system requirements: For some applications the target group itself is the entity, which is of interest. There is no need to deal with each single target as a separate entity. Advanced methods, which address this problem, may be found in [17], [18], [19]. The aim of this paper is the opposite one: An individual target tracking supplemented by group information. The integration of these group tracking aspects into the multidimensional data association context increase the individual tracking continuity and accuracy, and resolution capability significantly.

\section{Resolution Models}

The resolution of a radar system means the distance in any dimension between two targets so that they are reported as independent plots. It can be defined for all four radar components, whenever available: radar, azimuth, elevation and Doppler. Therefore, one has to find a heuristic model, which allows an overall approximation of such effects [16], [20].

\subsection{Linear Model}

The most simple class of models are the linear ones [16]. Let $w_{i}, i \in\{1, \ldots, L\}$ be the distance between the targets $t_{1}$ and $t_{2}$, e.g. in range azimuth, elevation or
Doppler and $d_{i}^{R}$ the sensor resolution. One postulates for the merging probability wrt. the component $\mathrm{i}$ :

$$
P_{i}^{M}\left(t_{1}, t_{2}\right)=\left\{\begin{array}{cl}
0 ; & \text { if } w_{i} \geq d_{i}^{R} \\
\frac{d_{i}^{R}-w_{i}}{d_{i}^{R}} ; & \text { if } w_{i}<d_{i}^{R}
\end{array}\right.
$$

The total merging probability is then

$$
P^{M}\left(t_{1}, t_{2}\right)=\prod_{i=1}^{L} P_{i}^{M}\left(t_{1}, t_{2}\right)
$$

\subsection{Gaussian Model}

W. Koch and G. van Keuk describe the merging by a Gaussian approach [14].

$$
P^{M}\left(t_{1}, t_{2}\right)=\exp \left(-\frac{1}{2} w^{\mathrm{T}} D w\right)
$$

This model takes care of the usually ellipsoidal resolution cells expressed by the matrix $D$.

\subsection{Resolution-Cell Model}

Another model developed in $[13,21]$ takes care of the standard deviation of the estimated distances:

$$
\begin{aligned}
& P_{i}^{M}\left(t_{1}, t_{2}\right)=\frac{1}{2}\left(\operatorname{erf}\left(\frac{w_{i}+d_{i}^{R}}{\sigma_{w_{i}}}\right)-\operatorname{erf}\left(\frac{w_{i}-d_{i}^{R}}{\sigma_{w_{i}}}\right)\right) \\
& +\frac{d_{i}}{2 d_{i}^{R}} \frac{1}{2}\left(\operatorname{erf}\left(\frac{w_{i}+d_{i}^{R}}{\sigma_{w_{i}}}\right)-\operatorname{erf}\left(\frac{w_{i}-d_{i}^{R}}{\sigma_{w_{i}}}\right)-2 \operatorname{erf}\left(\frac{w_{i}}{\sigma_{w_{i}}}\right)\right) \\
& +\frac{\sigma_{d_{i}}}{\sqrt{2 \pi} d_{i}^{R}}\left(\exp \left(-\frac{1}{2}\left(\frac{w_{i}+d_{i}^{R}}{\sigma_{w_{i}}}\right)^{2}\right)+\exp \left(-\frac{1}{2}\left(\frac{w_{i}-d_{i}^{R}}{\sigma_{w_{i}}}\right)^{2}\right)\right. \\
& \left.-2 \exp \left(-\frac{1}{2}\left(\frac{w_{i}}{\sigma_{w_{i}}}\right)^{2}\right)\right)
\end{aligned}
$$

with the settings

$$
\operatorname{erf}(x)=\sqrt{\frac{2}{\pi}} \int_{0}^{x} \exp \left(-\frac{u^{2}}{2}\right) d u
$$

\section{Filtering of clusters}

One has to distinguish between resolved and unresolved plot situations. The assumed dynamics of target $t$ is the constant velocity model. I.e., the prediction is defined by

$$
x_{k}^{t}=F_{k-1}^{t}\left(x_{k-1}^{t}\right)+q_{k-1}^{t}
$$


Here $x_{k}^{t}$ stands for the target state vector and $q_{k}^{t}$ describes a Gaussian process noise of covariance $Q_{k}^{t}$. The vector of a sensor measurement $j$ is written as $z_{k}^{j}$. This measurement is related to the actual target state by

$$
z_{k}^{j}=H_{k}^{t}\left(x_{k}^{t}\right)+r_{k}^{j}
$$

with a measurement noise $r_{k}^{j}$ defined as Gaussian distributed with covariance $R_{k}^{j}$.

\subsection{Resolved measurement}

In Kalman filtering one predicts the new state and covariance for target $t$ :

$$
\begin{gathered}
x_{k \mid k-1}^{t}=F_{k-1}^{t} x_{k-1}^{t} \\
P_{k \mid k-1}^{t}=F_{k-1}^{t} P_{k-1}^{t} F_{k-1}^{t}{ }^{\mathrm{T}}+Q_{k-1}^{t}
\end{gathered}
$$

These are projected into the measurement space:

$$
\begin{gathered}
z_{k \mid k-1}^{t}=H_{k}^{t} x_{k \mid k-1}^{t} \\
S_{k \mid k-1}^{t j}=H_{k}^{t} P_{k \mid k-1}^{t} H_{k}^{t}+R_{k}^{j}
\end{gathered}
$$

Finally, the Kalman gain matrix and the new state and covariance are calculated:

$$
\begin{gathered}
W_{k}^{t j}=P_{k \mid k-1}^{t} H_{k}^{t^{\mathrm{T}}} S_{k \mid k-1}^{t j}-1 \\
x_{k \mid k}^{t j}=x_{k \mid k-1}^{t}+W_{k}^{t j}\left(z_{k}^{j}-z_{k \mid k-1}^{t}\right) \\
P_{k \mid k}^{t j}=P_{k \mid k-1}^{t}-W_{k}^{t j} H_{k}^{t} P_{k \mid k-1}^{t}
\end{gathered}
$$

\subsection{Unresolved measurement}

One assumes, that plot $\mathrm{j}$ is unresolved and originates from track $t_{1}$ and $t_{2}$. We call this constellation a cluster of order two. The prediction step and projections of each individual track is the same as above. One assumes that some information of the signal strength $s_{k}^{t_{1}}$ and $s_{k}^{t_{2}}$ is given. One abbreviate

$$
\alpha_{k}^{t_{1}}=\frac{s_{k}^{t_{1}}}{s_{k}^{t_{1}}+s_{k}^{t_{2}}} \text { and } \alpha_{k}^{t_{2}}=\frac{s_{k}^{t_{2}}}{s_{k}^{t_{1}}+s_{k}^{t_{2}}}
$$

Taking the projection onto the measurement space into account the merging is

$$
\begin{gathered}
z_{k \mid k-1}^{t_{1} t_{2}}=\alpha_{k}^{t_{1}} z_{k \mid k-1}^{t_{1}}+\alpha_{k}^{t_{2}} z_{k \mid k-1}^{t_{2}} \\
S_{k \mid k-1}^{t j}=\alpha_{k}^{t_{1}{ }^{2}} H_{k}^{t_{1}} P_{k \mid k-1}^{t_{1}} H_{k}^{t_{1}{ }^{\mathrm{T}}} \\
+\alpha_{k}^{t_{2}{ }^{2}} H_{k}^{t_{2}} P_{k \mid k-1}^{t_{2}} H_{k}^{t_{2}}{ }^{\mathrm{T}}+R_{k}^{j}
\end{gathered}
$$

Finally, the Kalman gain matrix and the new state and covariance are calculated:

$$
\begin{gathered}
W_{k}^{t_{1} t_{2} j}=\alpha_{k}^{t_{1}} P_{k \mid k-1}^{t_{1}} H_{k}^{t_{1}} S_{k \mid k-1}^{t_{1} t_{2} j-1} \\
x_{k \mid k}^{t_{1} t_{2} j}=x_{k \mid k-1}^{t_{1} t_{2} j}+W_{k}^{t_{1} t_{2} j}\left(z_{k}^{j}-z_{k \mid k-1}^{t_{1} t_{2} j}\right) \\
P_{k \mid k}^{t_{1} t_{2} j}=P_{k \mid k-1}^{t_{1}}-\alpha_{k}^{t_{1}} W_{k}^{t_{1} t_{2} j} H P_{k \mid k-1}^{t_{1}}
\end{gathered}
$$

and similar for target $t_{2}$.

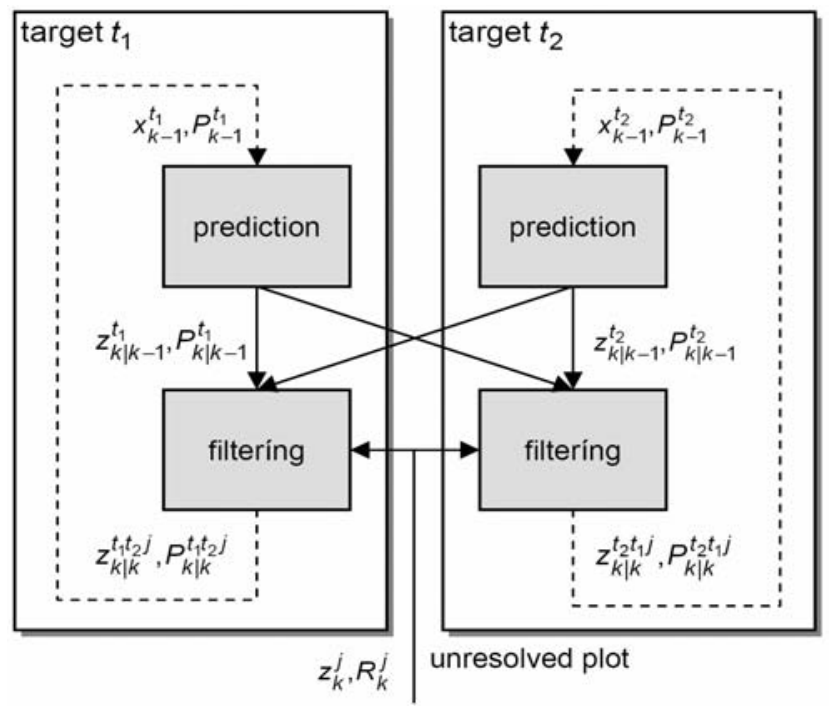

Figure 1. Filtering scheme for a cluster with an unresolved plot.

\subsection{The total update}

The new state for the track $t_{1}$ is defined by

$$
x_{k}^{t_{1}}= \begin{cases}x_{k \mid k}^{t_{1} j} & \text { single update with plot } j \\ x_{k \mid k}^{t_{1} t_{2} j} & \text { cluster update with } t_{2} \text { and plot } j\end{cases}
$$




$$
P_{k}^{t_{1}}= \begin{cases}P_{k \mid k}^{t_{1} j} & \text { single update with plot } j \\ P_{k \mid k}^{t_{1} t_{2} j} & \text { cluster update with } t_{2} \text { and plot } j\end{cases}
$$

\subsection{Possible modifications}

In $[13,21]$ a Gaussian approximation of a merge model is used, but therefore all covariances are assumed to be diagonal. Then the predictions in (16) and (17) obtain correction terms of the form:

$$
\begin{aligned}
& z_{k \mid k-1}^{t_{1} t_{2}} \rightarrow z_{k \mid k-1}^{t_{1} t_{2}} \\
& -U_{k \mid k-1}^{t_{1} t_{2}}\left(W_{k \mid k-1}^{t_{1} t_{2}}\right)^{-1} \exp \left(-\frac{1}{4} \operatorname{diag}\left(w_{k \mid k-1, i}^{t_{1} t_{2}} d_{i}^{r}\right)\right) w_{k \mid k-1}^{t_{1} t_{2}} \\
& S_{k \mid k-1}^{t j} \rightarrow S_{k \mid k-1}^{t j} \\
& -\left(U_{k \mid k-1}^{t_{1} t_{2}}\right)^{2}\left(W_{k \mid k-1}^{t_{1} t_{2}}\right)^{-1} \exp \left(-\frac{1}{2} \operatorname{diag}\left(w_{k \mid k-1, i}^{t_{1} t_{2}} d_{i}^{r}\right)\right)
\end{aligned}
$$

with:

$$
\begin{gathered}
U_{k \mid k-1}^{t_{1} t_{2}}=\alpha_{1}^{2} P_{k \mid k-1}^{t_{1}}-\left(1-\alpha_{2}^{2}\right) P_{k \mid k-1}^{t_{2}} \\
w_{k \mid k-1}^{t_{1} t_{2}}=z_{k \mid k-1}^{t_{1}}-z_{k \mid k-1}^{t_{2}} \\
W_{k \mid k-1}^{t_{1} t_{2}}=P_{k \mid k-1}^{t_{1}}+P_{k \mid k-1}^{t_{2}}
\end{gathered}
$$

Another modification is considered in [14]. Here the projected state $z_{k \mid k-1}^{t_{1} t_{2}}$ is augmented by $z_{k \mid k-1}^{t_{1}}-z_{k \mid k-1}^{t_{2}}$ and the measurement relation is enlarged :

$$
\left(\begin{array}{c}
z_{k}^{j} \\
w_{k}
\end{array}\right)=\left(\begin{array}{c}
H_{k}^{t_{1}}\left(\frac{1}{2} x_{k}^{t_{1}}\right)+H_{k}^{t_{2}}\left(\frac{1}{2} x_{k}^{t_{2}}\right)+r_{k}^{j} \\
H_{k}^{t_{1}}\left(x_{k}^{t_{1}}\right)-H_{k}^{t_{2}}\left(x_{k}^{t_{2}}\right)+q_{k}^{j}
\end{array}\right)
$$

where $w_{k}$ is assumed to be 0 and $q_{k}$ Gaussian distributed with covariance $D$.

\section{Data association with unresolved measurements}

The classical data association between tracks and plots is now extended to a data association between clusters and plots. The basic assumption for the association process is, that a plot of one scan is either

- $\quad$ resolved and

$\circ$ originates from one target or $\circ$ is a false alarm

- or is unresolved and belongs to a cluster of exactly two targets.

On the other side

- $\quad$ a track is updated by one (unresolved or resolved) plot of each scan or

- $\quad$ is not detected within a scan.

\subsection{Modification of 2 dimensional data association}

The established tracks are numbered by the set $\{1, \ldots, m\}$. The symbol 0 indicates the false plot hypothesis. To include the hypothesis of clusters consisting of two targets, one uses the following encoding:

$$
T^{2}:=T \times T, T=\{0, \ldots, m\}
$$

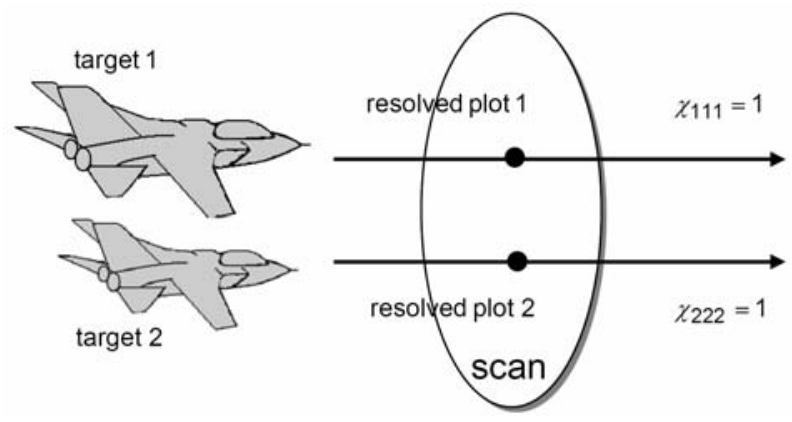

Figure 2. Resolved plot.

Here a combination $\left(t_{1}, t_{1}\right) \in T^{2}$ stands for a single track $t_{1}$, which is reported by a single resolved plot.

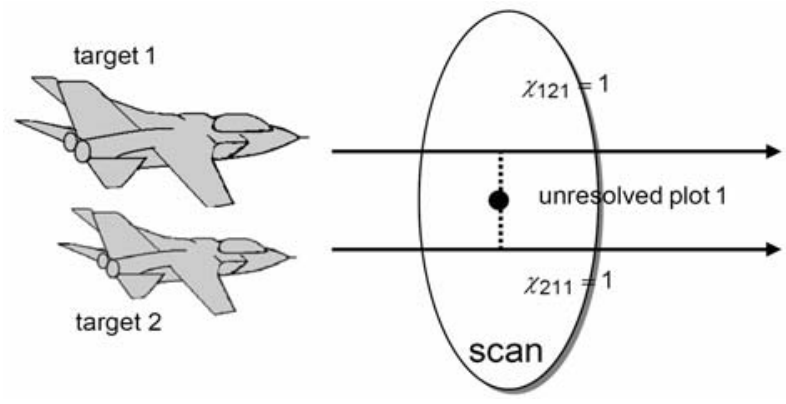

Figure 3. Unresolved plot.

A pair $\left(t_{1}, t_{2}\right)$ with $0 \neq t_{1} \neq t_{2} \neq 0$ determines track $t_{1}$, which is assumed in a 2-cluster with track $t_{2}$, and both are detected through an unresolved plot in the current scan. 
The symbol $(0,0)$ determines the false alarm hypothesis for a given plot. Finally, $\left(t_{1}, 0\right)$ and $\left(0, t_{1}\right)$ with $t_{1}, t_{2} \neq 0$ determines a track $t_{1}$, which falls into a 2-cluster with a false plot. This hypothesis will be ignored here.

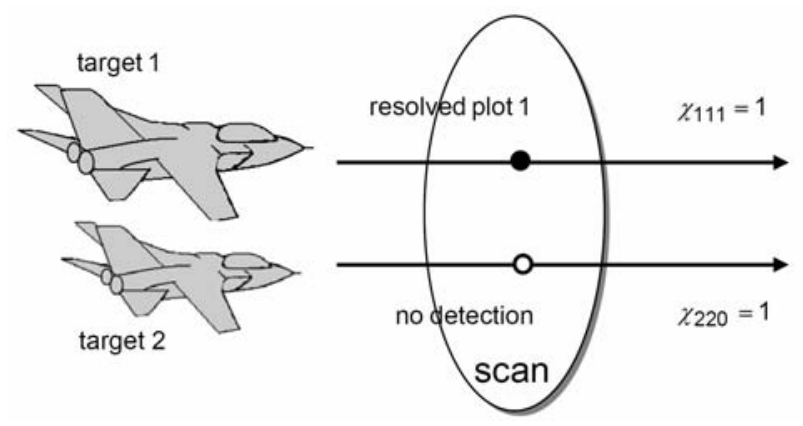

Figure 4. No detection.

The data association problem is now translated into an integer linear programming problem. Let $I_{1}=\left\{0, \ldots, n_{1}\right\}$ determine the (unresolved or resolved) measurements of the full further scan, where 0 symbolized a missed detection. One obtains the optimization problem:

$$
\min \sum_{t_{1}, t_{2}=0}^{m} \sum_{i_{1}=0}^{n_{1}} c_{t_{1} t_{2} i_{1}} \chi_{t_{1} t_{2} i_{1}}
$$

Here $\chi_{t_{1} t_{2} i_{1}} \in\{0,1\}$ is an indicator function, such that $\chi_{t_{1} t_{2} i_{1}}=1$ selects the association between the cluster $\left(t_{1}, t_{2}\right)$ and the measurement $i_{1}$. The $c_{t_{1} t_{2} i_{1}}$ are the negative logarithms of the association probabilities.

The indicator function is subject to the following constraints: The first constraint expresses the symmetry of the cluster definition. If target $t_{1}$ forms a cluster with target $t_{2}$ and has therefore a common unresolved plot $i_{1}$, then also target $t_{2}$ forms a cluster with target $t_{1}$ with common plot $i_{1}$, i.e.

$$
\chi_{t_{1} t_{2} i_{1}}=\chi_{t_{2} t_{1} i_{1}} ; t_{1}<t_{2} ; t_{1}, t_{2}=0, \ldots, m ; i=0, \ldots, n_{1}
$$

Each measurement is either unresolved and belongs to exactly two tracks, or is resolved and is a false alarm or belongs to a single track.:

$$
\sum_{t_{1}=0}^{m} \sum_{t_{2}=t_{1}}^{m} \chi_{t_{1} t_{2} i_{1}}=1 ; i_{1}=1, \ldots, n_{1}
$$

$$
\sum_{i_{1}=0}^{n_{1}} \sum_{t_{2}=0}^{m} \chi_{t_{1} t_{2} i_{1}}=1 ; t_{1}=1, \ldots, m
$$

Finally, some hypotheses are not considered, therefore their indicator is set to 0 .

$$
\begin{aligned}
& \chi_{000}=\chi_{t_{1} 0 i_{1}}=\chi_{0 t_{2} i_{1}}=0 ; \\
& t_{1}, t_{2}=1, \ldots, m ; i_{1}=0, \ldots, n_{1}
\end{aligned}
$$

The weight $c_{t_{1} t_{2} i_{1}}=-\ln \left(L_{t_{1} t_{2} i_{1}}\right)$ contributes to the cost of an overall association between clusters and plots. A regular update of a single track with a resolved plot resp. missed detection adds the following weight to the tracks score:

$$
\begin{aligned}
& L_{t_{1} t_{1} i_{1}}=\left[1-P_{D}\right]^{\delta_{i_{1}, 0}}\left[N\left(z_{k}^{i_{1}} ; z_{k \mid k-1}^{t_{1} i_{1}}, S_{k}^{t_{1} i_{1}}\right) \frac{P_{D}}{\rho_{F}}\right. \\
&\left.\prod_{\substack{j=1 \\
j \neq t_{1}}}^{m}\left(1-P_{M}\left(t_{1}, j\right)\right)\right]^{1-\delta_{i_{1}, 0}} ; \\
& t_{1} \in\{1, \ldots, m\}, i_{1} \in\left\{0, \ldots, n_{1}\right\}
\end{aligned}
$$

Finally, the weight for an unresolved plot is determined by:

$$
\begin{aligned}
& L_{t_{1} t_{2} i_{1}}=N\left(z_{k}^{i_{1}} ; z_{k \mid k-1}^{t_{1} t_{2} i_{1}}, S_{k}^{t_{1} t_{2} i_{1}}\right) \frac{P_{D}}{\rho_{F}} \\
& P_{M}\left(t_{1}, t_{2}\right) \prod_{\substack{j=1 \\
j \neq t_{1}, t_{2}}}^{m}\left(1-P_{M}\left(t_{1}, j\right)\right) ; \\
& t_{1}, t_{2} \in\{1, \ldots, m\}, i_{1} \in\left\{1, \ldots, n_{1}\right\}, t_{1} \neq t_{2}
\end{aligned}
$$

\subsection{Modification of 3 dimensional data association}

One is also able to modify the classical threedimensional data association, i.e. to consider two scans instead of one. Therefore, one uses indices of length 7 instead of 3 . The first 3 numbers characterise the track history up to the first scan. This track may be continued as part of a cluster of order 2 in the second scan. Therefore, the 4th up to the 6th number determines the track-history of the partner within the first scan. A repetition of the first 3 numbers codes a single track, which doesn't belong to a 2 -cluster in the second scan. Finally, the last number determines the measurement, which is associated in the second scan. To illustrate this, consider the formation in figure 5 .

Each track can be updated by at most one plot: 


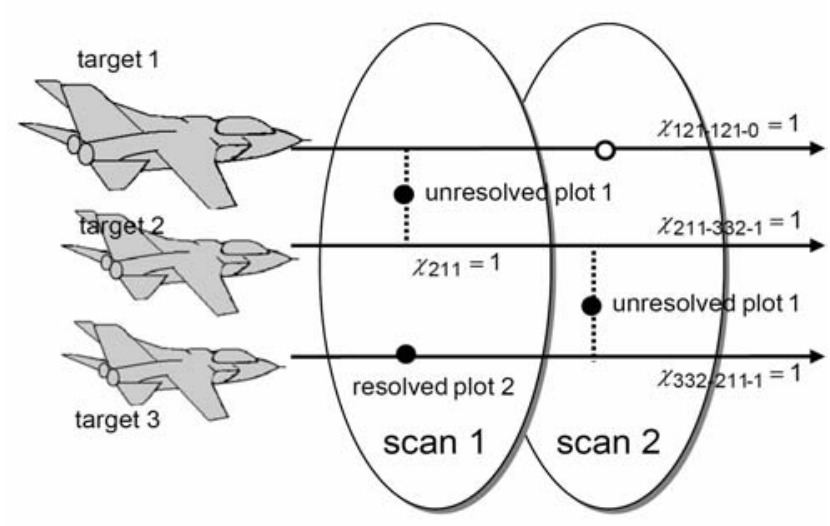

Figure 5. Association over two scans.

Targets 1 and 2 possess a common, unresolved plot in the first scan. Their trajectory is encoded as $\chi_{121}=1$ and $\chi_{211}=1$ up to this scan. Target 3 is unique reported through plot number 2, i.e. $\chi_{332}=1$. In the second scan, the first target is single, but not reported. I.e. $\chi_{1211210}=1$. The second and third target are clustered through the unresolved measurement 1 of scan number 2 . I.e. target 2 is given by $\chi_{2113321}=1$ and target 3 by $\chi_{3322111}=1$. In general one has to solve the optimization problem

$$
\min \sum_{t_{1}, t_{2}, t_{1}^{\prime}, t_{2}^{\prime}=0}^{m} \sum_{i_{1}, i_{1}^{\prime}=0}^{n_{1}} \sum_{i_{2}=0}^{n_{2}} c_{t_{1}} t_{2} i_{1} t_{1}^{\prime} t_{2} i_{1}^{\prime} i_{3} \chi_{t_{1} t_{2} i_{1}} t_{1}^{\prime} t_{2}^{\prime} i_{1}^{\prime} i_{2}
$$

subject to several constraints:

The first constraint expresses again the symmetry of the cluster definition:

$$
\begin{aligned}
& \chi_{t_{1} t_{2} i_{1}} t_{1}^{\prime} t_{2} i_{1}^{\prime} i_{2}=\chi_{t_{1}^{\prime} t_{2} i_{1}^{\prime} t_{1} t_{2} i_{1} i_{2}} ; \\
& t_{1}<t_{1}^{\prime} ; t_{1}, t_{2}, t_{1}^{\prime}, t_{2}^{\prime}=1, \ldots, m ; i_{1}, i_{1}^{\prime}=1, \ldots, n_{1} ; i_{2}=1, \ldots, n_{2}
\end{aligned}
$$

and

$$
\begin{aligned}
& \sum_{t_{1}^{\prime}, t_{2}^{\prime}=0}^{m} \sum_{i_{1}^{\prime}=0}^{n_{1}} \sum_{i_{2}^{\prime}=0}^{n_{2}} \chi_{t_{1} t_{2} i_{1} t_{1}^{\prime} t_{2} i_{1}^{\prime} i_{2}}= \\
& \sum_{t_{1}^{\prime}, t_{2}^{\prime}=0}^{m} \sum_{i_{1}^{\prime}=0}^{n_{1}} \sum_{i_{2}=0}^{n_{2}} \chi_{t_{1}^{\prime} t_{2} i_{1}^{\prime} t_{1} t_{2} i_{1} i_{2} ;} ; \\
& t_{1}<t_{2}, t_{1}, t_{2}=0, \ldots, m ; i_{1}=0, \ldots, n_{1}
\end{aligned}
$$

A plot of the second scan belongs to at most one track:

$$
\sum_{t_{1}, t_{2}, t_{1}^{\prime}, t_{2}^{\prime}=0}^{m} \sum_{t_{1}^{\prime}=t_{1}}^{m} \sum_{i_{1}, i_{1}^{\prime}=0}^{n_{1}} \chi_{t_{1} t_{2} i_{1}} t_{1}^{\prime} t_{2}^{\prime} i_{1}^{\prime} i_{2}=1 ; i_{2}=1, \ldots, n_{2}
$$

A plot of the first scan belongs to at most one track:

$$
\sum_{t_{1}, t_{1}^{\prime}, t_{2}^{\prime}=0}^{m} \sum_{t_{2}=t_{1}}^{m} \sum_{i_{1}^{\prime}=0}^{n_{1}} \sum_{i_{2}=0}^{n_{2}} \chi_{t_{1} t_{2} i_{1}} t_{t_{1}^{\prime} t_{2}^{\prime} i_{1}^{\prime} i_{2}}=1 ; i_{1}=1, \ldots, n_{1}
$$

A track is updated by at most one plot for each scan:

$$
\sum_{t_{2}, t_{1}^{\prime}, t_{2}^{\prime}=0}^{m} \sum_{i_{1}, i_{1}^{\prime}=0}^{n_{1}} \sum_{i_{2}=0}^{n_{2}} \chi_{t_{1} t_{2} i_{1}} t_{1}^{\prime} t_{2}^{\prime} i_{1}^{\prime} i_{2}=1 ; t_{1}=1, \ldots, m
$$

Finally, some hypotheses are not considered and their indicator is set to 0 .

$$
\begin{aligned}
& \chi_{0000000}=\chi_{t_{1} 0 i_{1} t_{1}^{\prime} t_{2} i_{1}^{\prime} i_{2}}=\chi_{0 t_{2} i_{1} t_{1}^{\prime} t_{2}^{\prime} i_{1}^{\prime} i_{2}=0 ;} \\
& t_{1}, t_{2}, t_{1}^{\prime}, t_{2}^{\prime}=1, \ldots, m ; i_{1}, i_{1}^{\prime}=0, \ldots, n_{1} ; i_{2}=0, \ldots, n_{2}
\end{aligned}
$$

The corresponding weighting coefficients are easy to generalize from the two dimensional data association above.

\subsection{The optimization problem}

Through the above treatment, the data association problem is transformed into an integer Linear Programming. There are several mathematical approaches which address this problem.

One of them is to preliminarily ignore the integer constraint $\chi_{t_{1} t_{2} i_{1}} \in\{0,1\}$ of the indicator function and use $0 \leq \chi_{t_{1} t_{2} i_{1}} \leq 1$ instead. Therefore, one is able to apply Linear Programming techniques. Especially the homogeneous self dual interior point method can be used to handle this problem, as proposed by [10]. The result $p_{t_{1} t_{2} i_{1}}$ is interpreted as pseudo probabilities similar to a JPDA approach but without suffering the track coalescence problem i.e.

$$
p_{t_{1} t_{2} i_{1}}=\operatorname{Prob}\left(\chi_{t_{1} t_{2} i_{1}}=1\right)
$$

This means $p_{t_{1} t_{2} i_{1}}$ is the probability that track $t_{1}$ forms a cluster with track $t_{2}$ with unresolved plot $i_{1}\left(t_{1} \neq t_{2}\right)$ resp. the probability that track $t_{1}$ is separated and is updated with the resolved plot $i_{1} \quad\left(t_{1}=t_{2}\right)$. This interpretation makes sense due to the constraints (31) (34). Similarly one argues for the 3-dimensional association. 


\section{Simulation Results}

To illustrate the performance of the described methods some evaluations of the two dimensional case are given below. For the solution of the optimisation steps the homogeneous self dual interior point method was used.

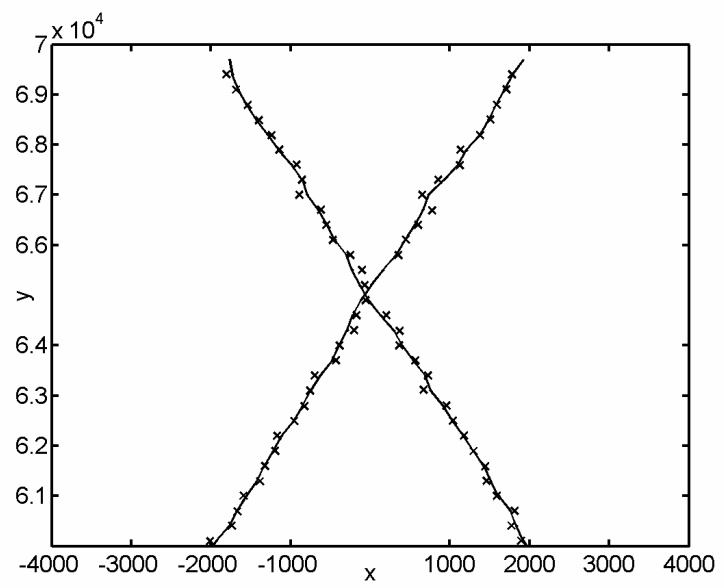

Figure 5. Two target cross scenario with single sensor.

Figure 5 shows a crossing scenario of two targets with unresolved measurements in the crossing area. processed by the modified two dimensional approach. One realizes, that both tracks are continuously tracked, even in this region.

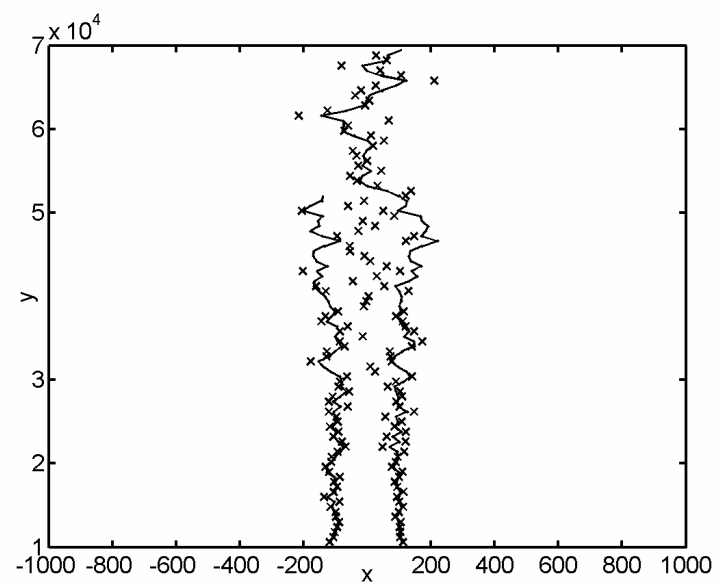

Figure 6. Two target split scenario with single sensor.

Figure 6 demonstrates two targets coming from outbound. This scenario is distinguished by the early track initialisation specifically in the region, where the sensor is not able to resolve the target situation continuously.

The figures 7 and 8 are about three target scenarios. Again one obtains continuous, accurate tracking, and early track initialisation capabilities even under unresolved plots. Whereas figure 7 considers the case of a single sensor, figure 8 is a multisensor scenario ("x"=sensor 1 , "+"=sensor 2). In this scenario radars with different resolution and accuracy behaviour are taken into account. One realises that for conventional tracking implementations, the worse sensor degrades the result of a plot level fusion. The proposed approached in this paper has the potential to solve this problem and ensures optimal continuous tracking and optimal resolution capabilities even for multi sensor tracking applications.

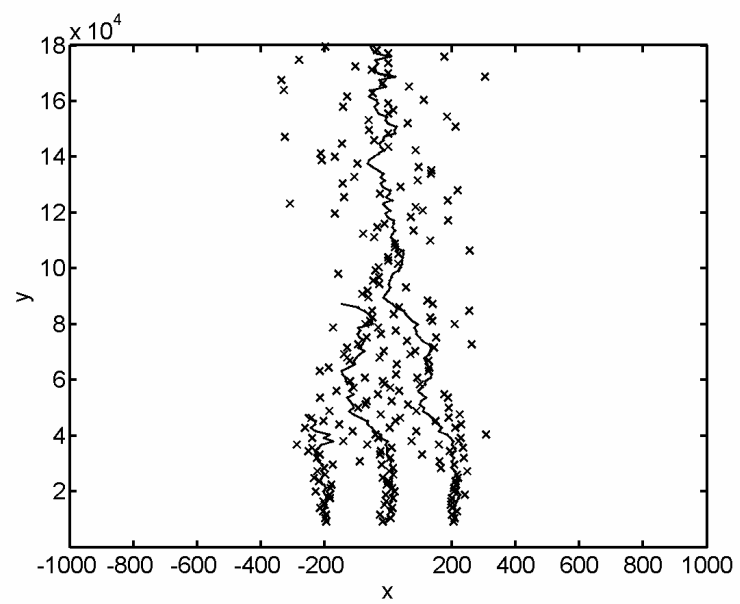

Figure 7. Three target split scenario with single sensor.

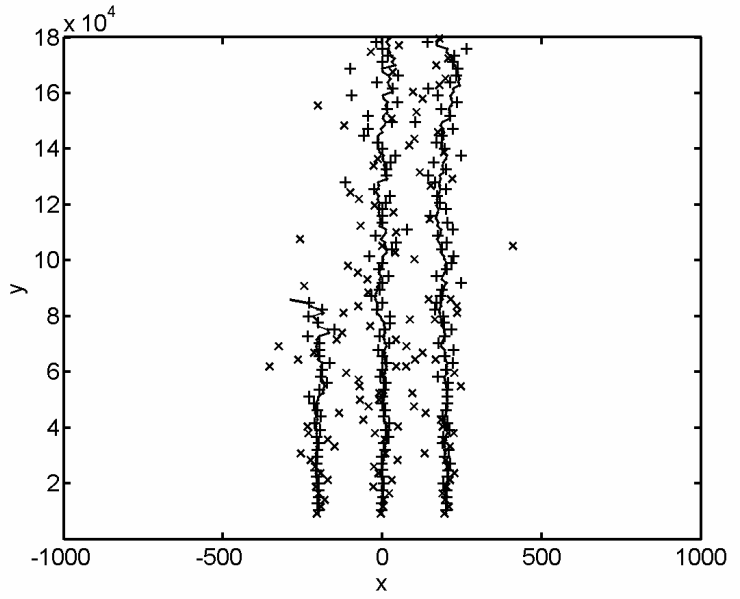

Figure 8. Three target spit scenario with two sensors.

\section{Conclusions}

A new algorithm is presented, which includes unresolved measurements and target clusters of order 2 into the multidimensional data association techniques. Also, a filter concept dealing with clusters is described. The presented simulation results show the applicability of the proposed new method. Especially, the two dimensional algorithm is very suited for dense target situations with a 
limited number of targets. It offers a significant improvement in track continuity, accuracy and initialisation capabilities while taking into account the real sensor phenomena of resolution conflicts.

\section{References}

[1] D. Bertsekas, Network Optimization - Continuous and discrete models, Athena Scientific, Belmont, Massachusetts, 1998.

[2] K. R. Pattipati, R. L. Popp and T. Kirubarajan, Survey of Assignment Techniques for Multitarget Tracking, in Multisensor Tracking - Applications and Advances Volumne III, Y. Bar-Shalom and W. D. Blair, Editors, Artech House, Norwood, 2000.

[3] R. Jonker and A. Volgenant, A Shortest Augmenting Path Algorithm for Dense and Sparse Linear Assignment Problems, J. Computing, vol. 38, pp 325-340, 1987.

[4] S. Blackman, Multiple Target Tracking with Radar Applications, Artech House, Norwood, 1986.

[5] A. B. Poore and N. Rijavec, Multi-target tracking and multi-dimensional assignment problems, SPIE Signal and Data Processing of Small Targets, Vol. 1481, pp 345356, 1991.

[6] A. B. Poore and N. Rijavec, A Lagrangian Relaxation Algorithm for Multi-dimensional Assignment Problems Arising from Multitarget Tracking, SIAM Journal on Optimization, Vol. 3, No. 3, pp 544-563, August 1993.

[7] F. Opitz: Data Association based on Lagrange Relaxation \& Convex Analysis, Symposium on Target Tracking and Sensor Data Fusion for Military Observation Systems, NATO R\&T, Budapest, October 2003.

[8] J.-B. Hiriart-Urruty and C. Lemaréchal, Convex Analysis and Minimization Algorithms I -Fundamentals, Grundlehren der mathematschen Wissenschaften 305, Springer-Verlag, Berlin Heidelberg, 1993.

[9] J.-B. Hiriart-Urruty and C. Lemaréchal, Convex Analysis and Minimization Algorithms II - Advanced Theory and Bundle Methods, Grundlehren der mathematschen Wissenschaften 306, Springer-Verlag, Berlin Heidelberg, 1993.

[10] X. Li, Z.-Q. Luo, K. M. Wong, E. Bossé, An Interior Point Linear Programming Approach to Two-Scan Data Association, IEEE Transactions on Aerospace and Electronic Systems, Vol. 35, No. 2, April 1999.
[11] S. Mizuno, M. J. Todd and Y. Ye, On Adaptive-Step Primal-Dual Interior-Point Algorithms for Linear Programming, Mathematics of Operations Research, Vol. 18, No. 4, November 1993.

[12] Y. Ye, M. J. Todd and S. Mizuno, $A n O(\sqrt{n L})$ Iteration Homogeneous and Self-Dual Linear Programming Algorithm, Mathematics of Operations Research, Vol. 19, No. 1, February 1994.

[13] K.-C. Chang., and Y. Bar-Shalom, Joint Probabilistic Data Association for Multitarget Tracking with Possibly Unresolved Measurements and Maneuvres, IEEE Transactions on Automatic Control, Vol 29, No. 7, pp. 585-594, July 1984.

[14] W. Koch, and G. van Keuk, Multiple Hypothesis Track Maintance with Possibly Unresolved Measurements, IEEE Transactions on Aerospace and Electronic Systems, Vol 33, No. 3, pp. 883-892, July 1997.

[15] H. Chen, T. Kirubarajan, Y. Bar-Shalom, Tracking of Spawing Targets with Multiple Finite Resolution Sensors, International Conf. on Information Fusion, Annapolis, MD, 2002, pp. 1511-1518.

[16] S. Blackman and Robert Popoli, Desing and Analysis of Modern Tracking Systems, Artech House, Boston, 1999.

[17] S. Gadaleta, A. Poore, S. Roberts, and B. J. Slocumb, Multi Hypothesis Clustering, Multi Frame Assignment, Tracking, Signal and Data Processing of Small Targets, edited by Oliver E. Drummond, Proceedings of SPIE Vol. 5428, 2004.

[18] E. P. Blasch, and T. Connare, Feature-Aided JBPDAF group tracking and classification using an IFFN sensor, SPIE Aerosense 4728, Signal and Data Processing of Small Targets, April 2002.

[19] E. P. Blasch and T. Connare, Group tracking of Occluded Targets, SPIE Int. Sym. on Aerospace/Defense Simulation \& Control, Conf 4365 - Acquisition and tracking XV, Orlando, FL, pp. 138-147, April 2001.

[20] M. Hamish, Modern Radar Systems, Artech House, Norwood, MA, 2001.

[21] Y. Bar-Shalom and X.-R. Li, MultitargetMultisensor Tracking: Principles and Techniques, YBS, Storrs, Connecticut, 1995. 